\title{
Wireless Mesh Networks for Infrastructure Deficient Areas
}

\author{
Roman Seibel, Nils-Hendrik Klann, Tim Waage, and Dieter Hogrefe \\ Georg August University of Göttingen \\ Computer Science Institute \& Faculty of Economics \\ 37077, Germany \\ seibel@cs.uni-goettingen.de, hhk@uni-goettingen.de, \\ tim.waagedstud.uni-goettingen.de, hogrefedcs.uni-goettingen.de
}

\begin{abstract}
Provision of internet access in infrastructure deficient areas is expected to bring profound economic and humanitarian benefit to developing countries. Notwithstanding, achieving this goal poses an economic and technical challenge. Due to technological and economical reasons cellular networks are regarded to be unable to deliver affordable distribution in the short term. Instead $802.11 \mathrm{~s}$ wireless mesh networking standard is identified to be a more viable and affordable partial solution to deliver broadband internet in the periphery of developing countries. A general focus is the individual incentive to participate in a mesh network rather than reliance on organisational bodies. This paper designs an architecture and business model for a low cost, low range distribution of internet access. The technical architecture is evaluated using simulation and is found to be viable, but demands optimisation.
\end{abstract}

Keywords: developing; ICT; cellular; 802.11s; mesh; business model; revenue sharing; simulation; capacity.

\section{Introduction}

This contribution takes a holistic approach to understand the peculiar situation of developing countries with regards to Internet access distribution and finds and evaluates a technical solution. We find that the barriers to internet provision in developing countries are of both economic as well as technical nature. Consequently we conceptualize on a new approach to distributing Internet access which combines theories from the fields of economics and computer science to tackle the above mentioned challenges. Holistic also refers to the fact that prior to finding yet another technical solution, an economic and technical perspective is applied to determine the solutions requirements. Following this the technical solutions and economic rules, in terms of a business model, are designed. Eventually the solution is being technically evaluated.

\subsection{Economic Perspective}

Affordable access to reliable Information and Communication Technologies (ICT) poses a key element to economic growth, human development and social progress of 
societies. Following the argument of [1] the broader availability of computers as well as information and communication technology has been recognized as a major contributor to the growth experience of industrialized countries during the 20th century. In quantitative terms the growth-enhancing effect attributable to the increased availability of ICT in industrialized countries is estimated to amount to as much as one additional percentage point of annual growth for countries such as the United States, Great Britain or Germany. [2] An additional percentage in growth has a non negligible impact over time, for it is exponential by nature.

Unfortunately access to this technology and thus its inherent advantages is distributed very unevenly around the world - a fact often described as the "digital divide" [3], [4]. As access to ICT infrastructure remains asymmetrically distributed on a global scale, so are the advancements. Societies and individuals are potentially able to reap from technologies such as broadband internet and mobile communications.

Much like in the case of the industrialized countries the availability of ICT is expected to act as a stimulant to economic growth in developing countries. As noted in [4] an average increase of 10 percent in mobile phone penetration in a developing country is expected to boost economic growth by around 0.81 percent annually. A similar increase in broadband penetration is expected to have an even higher growth effect of 1.4 percent annually. In addition to the issue of economic growth alone, the importance of ICT technology like "road, rail or electricity." infrastructure, is to an increasing extent recognized as an equally important pre-condition for human development [5]. In a similar vein broad provision of ICT infrastructure is identified as an important target within the Millennium Development goals, where an increase in the access to telephones, cellular networks and broadband internet is seen as a crucial development target.

The gravity created by a lack of access is most visible when contrasted with examples where individuals in these countries were able to make use of ICT. One important aspect is the provision of easy access to market information. [6] and [7] provide illustrative examples for the cases of India and Niger where the new availability of market information through mobile phones created major increases in the wellbeing of society. Other benefits include the improved operation of health services, schools and government services [5], [8], [9] The positive linkage between access to information and human development is further elaborated in [10].Although the positive impact of a reliable and affordable access to ICT services is by now beyond doubt, less clarity exists in concepts aiming at the actual delivery of such services to individuals in poor countries. Efforts have clearly been undertaken in connecting countries - for example on the African continent via undersea cables with the Internet. [11] This represents a crucial step towards achieving indiscriminate access to ICT services for as many individuals as possible, yet falls short of a complete solution. Obviously the sole establishment of a network connection through an undersea fiber represents a useful but also potentially insufficient measure if not combined with a solution to disseminate access to this connection throughout the entire country. As an isolated measure alone achieving an undersea wire without having a dissemination strategy only serves for replicating the between-country digital divide within the borders of the same country thus creating a within-country gap in ICT access. Instead a framework is required which provides solutions helping to overcome both the technical as well as economical obstacles, policy makers and 
enterprises face when extending access to ICT from the shorelines of countries to doorsteps of the actual users.

If we accept the fact that network connection is becoming viable on the country level the focus of attention has to be concentrated on solving downstream part of the provision challenge which describes the establishment of ICT services on a more local village or household level. We identify the following requirements for a distribution concept.

- Low regulatory effort for spectrum licensing.

- Low reliance on large organizational bodies.

- Simple entrepreneurial business model, with individual incentives.

- Low cost, mass market equipment availability.

In the discussion around this topic it is generally assumed that persisting structures on developing countries provide no practicable environment for wired-based approaches as usually seen in industrialized countries. Last have the luxury of a dense telephony cable network, installed in the previous century, suitable for broadband access distribution. Further, as [12] points out, even if installed, earth wire solutions are extremely prone to cable theft as the copper material used in the process represents a significant revenue source for delinquents. Since cellular networks become more ubiquitous globally, a typical suggestion to bridge the so-called "last mile" between an internet provider and the actual household relies on cellular networks. Basically the argument is that already existing mobile phone technology simply needs to be upgraded to data service capability to provide mobile internet access throughout society. We provide some discussion in the upcoming chapter and argue against cellular networks and pro 802.11 mesh, which is fulfilling the above mentioned requirements for a provision of ICT services at the provincial, village and even urban level.

\subsection{Cellular Unfitness}

Historically GSM (2G) based networks have expanded rapidly in developing areas, offering low cost voice services and slow high cost data services based on GPRS or EDGE. These networks can be upgraded to WCDMA networks to facilitate broadband data services, that can offer up to $7,2^{1} \mathrm{Mbps}$ with HSDPA, a path that has been taken in developed countries during the last decade. Further $3 \mathrm{G}$ hardware production has gained a large momentum on world markets and economies of scale allow manufacturers to produce low cost equipment, suitable for low income countries.

As we have discussed in the previous section, developing areas lack of infrastructure that could support access distribution on household level. Facilities such as telephone-, television- or even sufficient electric grid cable work is a scarce good. It is natural to assume that cellular networks can also be used for the last mile in developing countries, to offer Internet access to consumers and small businesses. However we argue that $3 \mathrm{G}$ cellular networks have certain limitations, that will render them an expensive solution, which can only serve a small percentage of the population, mainly high income households and larger enterprises.

\footnotetext{
${ }^{1}$ HSDPA category 8, using QPSK and 16-QAM modulation with up to 10 codes.
} 
With cellular architecture Base Station Transceivers (BTS) are set up in a certain location with a certain radio coverage radius of a few hundred meters to a few kilometers (depending on parameters like transmit power, antenna design, frequency and environmental obstacles). This area, the cell is used to provide clients such as handsets and cellular modems with $2 \mathrm{G} / 3 \mathrm{G}$ voice and data services. It makes sense to cover greater developing areas with so called macro cells, to reach as many customers as possible, while investing in as few cell sites as feasible.

The problem with WCDMA as multiple access technology is that its effective cell capacity, i.e. the maximum number of clients it can serve, is interference limited [13]. This is due to inter symbol interference (ISI) which implies that actual broadband cellular data services are only available to clients if a) they are located near the cells core with a reasonable signal quality and $b$ ) the number of simultaneous clients using the cell is small, which results in minimal interference. Nevertheless this fact is contradicting with the macro cell architecture mentioned above and leads to a low effective data rate and reduced effective coverage radius. A low effective radius results in the necessity for a higher cell site density which drives cost and therefore higher price, a property unwanted in low income countries. Currently developed countries counter measure this effect with the introduction of femto cells. Femto cells are small, low power cells, installed on customer premises with IP based backhaul connection to a cellular network operators core network. The IP based backhaul is usually realized via a separate cable, DSL or fiber broadband access of the customer. As a result macro cell load decreases, since the majority of data intensive services are used indoors, mostly at home. This effectively reduces the number of users per macro cell, decreasing interference and augmenting the macro cells effective radius. It can be argued that this solution extends WCDMA to a universal technology, that serves both, high performance data service needs indoors and seamless broadband data services while mobile. Notice, that developing areas generally do not have any kind of infrastructure that could support femto cell backhaul.

As a result, 3G networks, based on WCDMA cannot serve as the technology of choice to provide access to low and middle income households. Among other a key disadvantage of $3 \mathrm{G}$ cellular networks are the associated regulatory issues as well as the high costs associated with the service. As [14] points out $3 \mathrm{G}$ cellular networks are based on a vertical and centralized business framework with which users solely act as clients without much possibilities of actually participating in the provision process. Furthermore internet provision through $3 \mathrm{G}$ infrastructure faces considerable technical and legislative challenges in developing countries. As $3 \mathrm{G}$ services rely on a fixed spectrum the technology becomes vulnerable to issues such as spectrum scarcity on the one hand as well as insufficient spectrum management by government agencies and potentially ICT providers [15]. As [16] points out licenses to use bandwidth associated with a $3 \mathrm{G}$ cellular network structure can imply considerable costs for companies wishing to enter the market.

Long Term Evolution (LTE), successor of WCDMA networks uses Orthogonal Frequency Division Multiple Access (OFDMA) for downlink. It reduces the interference problem that occurs with WCDMA networks, by assigning individual, quasi non overlapping sub channels to clients. It needs to be analyzed how well LTE could be used in developing countries for the last mile, regarding performance and capacity. Although LTE is an interesting alternative, but it underlies the same 
economic and regulatory constraints as $3 \mathrm{G}$ networks and it might take a decade or more until LTE reaches economies of scale in order to be able to be rolled out in the majority of developing countries.

At large cellular networks do not fit the above mentioned requirements. Spectrum is licensed and tends to be expensive, especially if auctioned amongst multinational telecommunication companies. A large organization is necessary to operate a cellular network and equipment such as base stations, controllers and end user modems are not as low cost as alternatives. As opposed to this 802.11 networks provide more room for innovative concepts given their "decentralized, bottom-up" structure. Low regulatory effort through usage of Industrial Medical Scientific (ISM) and Unlicensed National Information Infrastructure (UNII) spectrum is possible. The fact that individuals can easily buy and install standard equipment secures low reliance on organizational bodies such as mobile network operators. It is further possible to implement simple entrepreneurial business models as will be discussed shortly. Finally low cost mass market equipment makes it affordable even for lower income households. Further low cost mesh routers offered by [17] are suitable for the supplier side of the market, whereas low cost computers such as netbooks and tablet PCs equipped with 802.11 Wireless LAN interfaces are a fit for clients, i.e. demand side of the market.

We have now discussed the ICT situation of developing countries and how access to Internet services can have a significant positive economic and humanitarian impact. We have established key properties for a technical and economic solution and introduced and criticized cellular networks as an obvious but problematic approach. We will now introduce the concept of wireless mesh networks, based on afore mentioned IEEE 802.11 standard, its architecture, a business model and capacity simulations, in order to consider it as a further alternative for infrastructure deficient areas for household level access distribution.

\section{Wireless Mesh}

This section describes the architecture of a 802.11 based wireless mesh network for the last mile of infrastructure deficient areas, followed by a business model and simulation results of the architectures capacity in worst case/peak period scenarios.

\subsection{Architecture}

Fig. 1 depicts 9 elements which are being introduced from left to right.

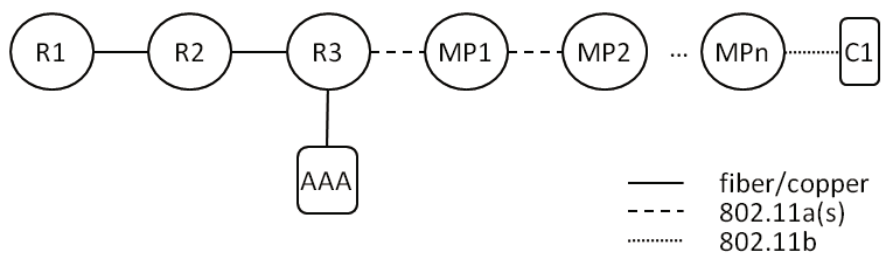

Fig. 1. 
The left most element shows a router R1, belonging to a Tier 1 Internet Service Provider (ISP). Tier 1 ISPs voluntarily or commercially peer with each other and thus establish the backbone of the global Internetwork. Tier 1 uses broad band fiber- and copper cables to interconnect routers. Tier 2 router R2 is connected to Tier 1 via broad band infrastructure. Tier 2 ISP is customer of Tier 1, it resells Internet access to individual customers termed Tier 3, which can be end customer or a further reseller i.e. ISP. R3 is connected to R2 via last mile copper cable or radio channel (DSL, 802.16, WCDMA, Satellite).

Tier 3 maintains an Authentication, Authorization and Accounting (AAA) entity, interconnected with R3. Mesh Points (MPs) are routers with a wireless 802.11a (5 Ghz) interface, that uses mesh mode (802.11s) to interconnect with each other and establish a mesh backhaul. MPs carry a second interface based on $802.11 \mathrm{~b} / \mathrm{g} / \mathrm{n}(2,4$ $\mathrm{Ghz}$ ) to connect to client devices, here $\mathrm{C} 1$. We separate the client radio access from the mesh backbone to reduce interference. We choose ISM 2,4 Ghz band for the client domain, because it is the most ubiquitous interface in end devices und more robust and far reaching, since it uses a lower frequency compared to 802.11a. We identify the $5 \mathrm{Ghz}$ band as less crowded by other technologies (Bluetooth, cordless phones, microwave ovens, etc.). Also its lower spatial coverage, due to the higher frequency, is less relevant, because in multi hop architectures, distance between nodes can be relatively low, compared to single hop architectures. Notice: In case $802.11 \mathrm{n}$ is used for the client spectrum domain, only single band $(2,4 \mathrm{Ghz})$ mode should be used, to prevent interferences with $802.11 \mathrm{a}(5 \mathrm{Ghz})$.

\subsection{Business Model}

The broad aim of every technological innovation is to realize some sort of benefit or value to participating parties. For end users the benefit is Internet access and for providers it is economic benefit of financial transactions made for that service by end users. In order to describe how the logic of value creation and capturing is conducted with this particular technology, we use a simplistic business model, described by a value chain and accounting relationship model. This business model focuses on two afore mentioned requirements of low organizational dependence and simple entrepreneurial incentives.

Fig. 2 introduces following entities of the value chain from left to right. Tier 2 ISP purchases access form Tier 1 and resells it to Tier 3. Tier 3 purchases Internet access from Tier 2 and resells it to Clients. Tier 3 can be a subsidiary of Tier 2 or an independent individual. The revenue earned by Tier 3 is shared with MP maintainers that evidently participate in routing of traffic to and from an associated client.

MP maintainers are individuals in contractual relationship with Tier 3.

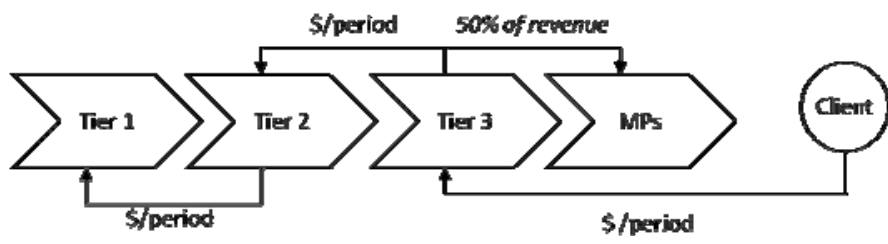

Fig. 2. 
The accounting relationship is the following. Client 1 purchases credit with Tier 3 via micro payment for a certain amount of US\$, per certain period, e.g. days. Notice this example uses (mobile) micro payment ${ }^{2}$ rather than cash exchange. This allows simple automated and secure transactions. The situation in developing countries shows a widespread adoption of GSM based micro payment systems, due to lack of banking services for various economic reasons. The client uses the credit and generates traffic via a first hop to an access point and several following hops via wireless mesh backhaul. After the period has passed, MP1, .. MPn report to AAA entity their routing/forwarding effort for Client 1. AAA verifies reports, calculates respective share for each MP and transfers a certain amount of currency to $\mathrm{MP}$ maintainer via micro payment.

The architecture described is standard mass market equipment. The used protocols on 802.11 networks such as hybrid wireless mesh protocol (HWMP) studied and suitable, if still not optimal for this scenario as will be shown in the next section. The innovation of this approach is not the technical refinement, but the combination of affordable mass market equipment and a business model, that applies micro economic incentives to individuals, in order to motivate the participation in a wireless mesh network in vicinity to a broad band gateway. Thus meeting above requirements, the solution capitalizes not only on low organizational participation and entrepreneurial incentives but also on low cost mass market products and low regulatory effort.

\subsection{Example Scenario}

The following describes an example ISP Tier 3 sets up a router, connected to Tier 2 and a AAA element in a urban or rural area. This individual is most likely someone already reselling internet access in his internet café/shop. Neighboring individuals (MP maintainer) of Tier 3 premises obtain mesh points and install and associate them with router and AAA element of Tier 3 via 802.11a (mesh mode). Micro payment details are shared between Tier 3 and MP maintainer. The service is setup and ready to use by clients. A user (Client1) detects a wireless network, based on 802.11b in the vicinity of a MP associated to Tier 3. Client 1 associates to MP and is greeted with a captive portal of Tier3. Payment instructions are presented for a pass for a certain period, e.g. 24 hours for $1 \mathrm{US} \$$. Client 1 uses micro payment to transfer 1 US\$ to Tier3's mobile payment account. The client no uses the Internet connection realized by Tier 3 and cooperating MP maintainers for 24 hours and generates traffic along certain mesh path(s). At the end of 24 hours, MP maintainer receive a share of for example $50 \%$ of the revenue, i.e. 50 US. Each MP maintainer receives a portion of this share according to performed forwarding effort on his mobile payment account. For example if three MP were involved MP1, MP2 and MP3 and all MPs have contributed the same amount of data forwarded to and from Client 1, the share will be distributed equally, 16.66US $\varnothing$ per MP. Notice: This will be the case in a mesh scenario, with static routing topology throughout the alleged period, but will vary if routing topology changes.

One consequence of this revenue sharing model needs to be mentioned. If the topology is, as shown in Fig.3, with R3 as the root node and a hierarchical arrangement

\footnotetext{
${ }^{2}$ See M-PESA www.safaricom.co.ke, www.roshan.af, www.vodacom.co.tz
} 
of MPs around it, there will be sets or rings of MPs closer to R3 and more distant. We classify those nodes with hierarchy number tuples such as MP1.2, which means Mesh Point ring 1, number 2. MP1.2 which is closer to R3 will play a greater role in traffic forwarding for clients than MP2.1, since also MP2.2 and MP2.3 are associated to it with its clients. Clients are denominated $\mathrm{C} 1, \mathrm{C} 2 \ldots \mathrm{Cn}$. Hence MP1.2 is crucial to the functioning of a significant part of the network. Since its indispensability it makes sense that MP1.2 will receive greater revenue shares for forwarding effort. The greater the number of clients in outer rings, the greater the income for MPs in inner rings will be relative to the income of MPs in more distant rings. This creates an incentive for MP maintainers in crucial positions to keep MPs active and reachable by other nodes. This may include the necessity to provide a stable power source such as grid, batteries, wind or solar power. Notice: Also MPs in inner rings have associated clients, these are left out of the diagram for simplicity.

\subsection{Capacity Simulation}

The following section describes the simulation setup produced with Qualnet simulator[18], a commercial derivate of GloMoSim[19]. We used Qualnet 5.0, Wireless Library, with an implementation of IEEE P802.11s/D1.00 draft standard. 802.11s uses a hybrid path selection protocol on medium access (MAC) layer, which means that either on demand or tree based modes are supported. In order to analyze the capacity of the architecture and evaluate the viability of the set up and business model, the following two scenarios are being created. One small area scenario to test general short distance two hop access to a nearby Internet gateway and one large area scenario to test for upper boundaries of the topology.

Fig.3 shows 36 Mesh Points (MPs) that are placed around a Mesh Portal Point (MPP), that has a $10 \mathrm{Mbps}$ backhaul to a Tier 2 ISP. The distance between two neighbor MPs is $100 \mathrm{~m}$. Thus the mesh backhaul radius is $300 \mathrm{~m}$. This covers an area of $282.600 \mathrm{~m}^{2}$ (area of circle) with equal MP density. MPs are interconnected with 802.11a on UNII 5Ghz band. The figure shows only MP1.1-1.6 denominated and all "leaves" of MP1.2s connection tree, in order to simplify the figure. All MPs are attached to MPP in the same manner. Each MP provides access to a certain number of clients via 802.11b ISM 2,4 Ghz band. All dataflow is directed to and from the central MPP. Tier 2 Router. Tables below show the actual average throughput per client in case every client produces a constant bit rate (CBR) of $64 \mathrm{kbps}$ between itself and Tier 2 Router. Although in practice website traffic is more arbitrary and generates high bit rate bursts, we use CBR to create a steady and controlled load, to provoke the topology and used protocols to reveal its limitations. We use $64 \mathrm{kbps}$ rather than a more realistic $1 \mathrm{Mbps}$ per client, to prove if it is possible to establish a data service that allows basic applications such as email and web browsing. These scenarios are supposed to simulate peak time traffic with all clients active at the same time. It can be assumed that a single clients connection will perform better most of the time.

The first simulation uses MP1.1-1.6" the number of clients per MP varies from 4 to up to 32 . We aim to investigate the maximal number of clients that can obtain a reasonable quality of service, near to an Internet gateway. The data flows over two hops, one from client to MP and second from MP to MPP. Table 1 shows results with 


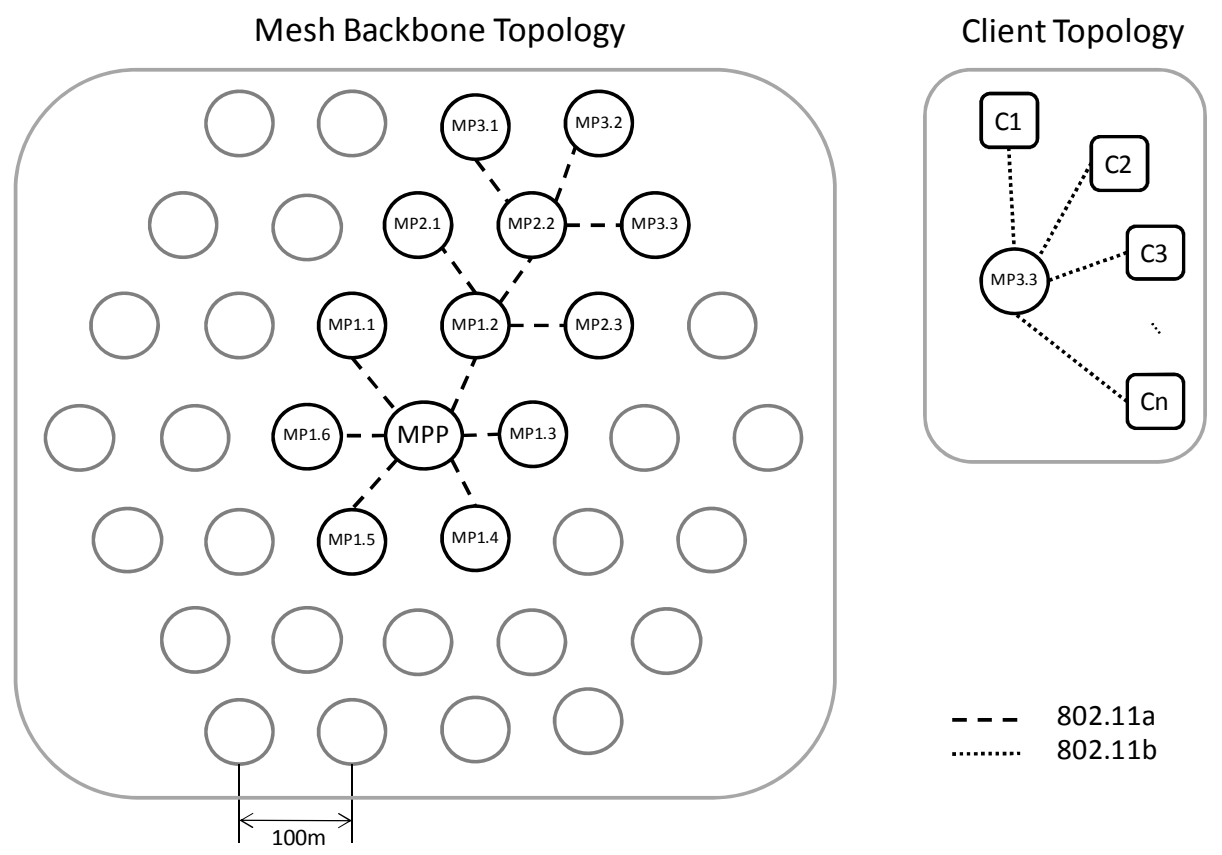

Fig. 3 .

average throughput per client in kbps. Because performance per client varied, variation is captured as the standard deviation and presented in parentheses. The lower the value is in relation to throughput, the more equal or fair network bandwidth is shared.

Table 1.

\begin{tabular}{llllll}
\hline $\begin{array}{l}\text { clients per } \\
\text { MP }\end{array}$ & $\begin{array}{l}\text { Total number of } \\
\text { clients }\end{array}$ & \multicolumn{4}{l}{ Avg. Throughput (standard deviation) [kbps] } \\
\hline 4 & 24 & 64.40 & $(00.08 \bumpeq 0 \%)$ & 64.24 & $(00.00 \triangleq 0 \%)$ \\
8 & 48 & 59.36 & $(04.80 \bumpeq 8 \%)$ & 64.24 & $(00.00 \triangleq 0 \%)$ \\
16 & 96 & 33.44 & $(21.44 \bumpeq 64 \%)$ & 40.56 & $(30.96 \bumpeq 76 \%)$ \\
32 & 192 & 23.44 & $(25.60 \bumpeq 109 \%)$ & 20.08 & $(29.44 \bumpeq 146 \%)$ \\
\hline
\end{tabular}

It is possible to provide fair and reliable Internet access at a rate of ca. 60kbps for 48 clients in a dense area around the MPP. It is further possible to deliver 30-40 kbps with high deviation of $60-70 \%$ to 96 clients. More clients will augment the deviation up to $100 \%$ of the average throughput and render the service useless for some clients. From this initial simulation experiment we speculate that a single broadband connection can provide reasonable low bandwidth Internet access to clients in the vicinity of two hops for 50-100 clients. We assume that establishing a six MP topology, with up to 100 clients combined with traffic shaping of $128 \mathrm{kbps}$ or 256 
kbps per client and a more realistic arbitrary traffic behavior could deliver viable low cost quality of data service on household level.

Under the simple assumption that one household covers about $500 \mathrm{~m}^{2}$ (small house), the area in the first scenario could accommodate about 60 households $\left(31.400 \mathrm{~m}^{2}\right.$ with $100 \mathrm{~m}$ radius), one access per household is therefore feasible. If such Internet gateways, owned by Internet shops, private higher income individuals or others were densely available, the six MP, two hop topology would solve a significant part of the last mile connection problem. But in reality density of broadband infrastructure is low and therefore more hops are required to serve more clients not close to the infrastructure. In our second experiment we investigate how well the technology scales in terms of increased hop counts.

The second simulation uses all 36 MPs covering the complete area. Clients use two to four wireless hops to and from the MPP. As Table 2 displays the bandwidth equality amongst clients is less, compared to the previous scenario. Standard deviation for only 36 simultaneous clients amounts $50 \%$ of the average throughput for TCP and about $70 \%$ for 72 clients. We observe that in larger networks the inner nodes often achieve much higher throughput than outer nodes. Therefore fairness mechanisms could be established in order to provide equal service all over the network. The comparison of UDP to TCP reveals that fairness is a greater issue for TCP.

Table 2.

\begin{tabular}{|c|c|c|c|c|c|}
\hline \multirow{2}{*}{$\begin{array}{l}\text { clients per } \\
\text { MP }\end{array}$} & \multirow{2}{*}{$\begin{array}{l}\text { Total number of } \\
\text { clients }\end{array}$} & \multicolumn{4}{|c|}{ Avg. Throughput (standard deviation) [kbps] } \\
\hline & & \multicolumn{2}{|c|}{ TCP traffic } & \multicolumn{2}{|c|}{ UDP Traffic } \\
\hline 1 & 36 & 48.48 & $(24.88 \wedge 51 \%)$ & 46.96 & $(03.44 \triangleq 7 \%)$ \\
\hline 2 & 72 & 29.04 & $(20.26 \triangleq 69 \%)$ & 30.80 & $(14.32 \triangleq 62 \%)$ \\
\hline 3 & 108 & 24.80 & $(24.32 \wedge 98 \%)$ & 15.20 & $(08.00 \triangleq 52 \%)$ \\
\hline 4 & 144 & 17.68 & $(25.04 \bumpeq 141 \%)$ & 12.48 & $(08.08 \bumpeq 64 \%)$ \\
\hline
\end{tabular}

Considering our household example above, this topology could fit 500 households $\left(282.600 \mathrm{~m}^{2}, 300 \mathrm{~m}\right.$ radius), but less than 100 clients (one out of five ) can be served a reasonable Internet access. Hence the D1.00 802.11s mesh networks scalability as implemented in the simulator is low.

This scenario shows that for large multi hop networks, the wireless mesh topology and protocols will be a significant bottleneck in peak periods and can only serve a low overall number of clients. Here further investigations of protocols and measures of fairness need to be conducted.

\section{Related Work}

A framework of a multitude of wireless technologies for infrastructure deficient areas is provided by [20]. There exist several projects implementing urban and rural WMNs for city and communal population [21-22], but none of which implements incentive systems for individual participants. These networks are either for free use or for commercial use, financed and maintained by a third party. 
A micropayment scheme for a captive portal for wireless internet access is designed and implemented by [23]. The concept is based on premium Short Message System (SMS) messages, it is not useable with current mobile payment schemes such as MPESA[24].

The capacity or scaling capability with increased hop count is identified as the main challenge of $802.11 \mathrm{~s}$ as a possible last mile technology. The following related work gives an insight into possible solutions and further challenges.

As [25] point out, the medium access scheme of $802.11 \mathrm{~s}$ is based on traditional 802.11 MAC protocol Enhanced Distributed Channel Access (EDCA), which is designed for 1 hop scenarios and therefore is not a mesh aware access scheme. [26] analyses the general capacity problem of wireless mesh topologies. They apply a concept called bottle neck collision domain to identify a link, that creates a bottleneck for the whole topology, considering load of all involved links affected by the medium access scheme. Usually one of the links between MPP an MP1.1-1.6 constitutes a bottleneck.

Several alternative [27] schemes exist, such as Mesh Deterministic Access (MDA) an optional 802.11s scheme or Mesh Network Alliance (MNA) scheme. The latter applies the concept of segregating mesh backhaul traffic from client access traffic in contention and non contention periods and spatial frequency reuse, by considering signal strength of neighbor nodes. The former uses a distributed time division access scheme. Both schemes try to reduce the unnecessary delay of multihop mesh packets, which leads to low scalability as we have encountered. A further method of increasing scalability is the employment of multiple channels for mesh backhaul traffic, thus reducing the bottleneck collision domain significantly as proposed in [28].

\section{Summary and Future Work}

In this paper we have analyzed developing markets from the economic point of view and pointed out the immense economic and humanitarian benefits of a broader distribution of Internet access in infrastructure deficient areas. We have identified requirements for a solution that approaches low cost, low regulation and entrepreneurial properties. Further we have designed a solution that based on 802.11 s can bridge the "last mile" to low income households on a short range. The solution implements low cost equipment paired with a revenue sharing business model that promises rapid decentralized deployment. We have finally evaluated the capacity of the architecture and found it to be viable for certain scenarios, with demand for further optimization. Future works on this concept may cover open architectural questions and optimization investigations, such as collected below.

- $\quad$ Exact AAA architecture: designed, implementation and evaluation regarding performance and security.

- Security analysis for mesh backbone and client association with regard to authentication and encryption is necessary.

- The possibility of roaming between different Tier 3 networks or administrative domains: investigation and incorporation into business model and architecture design. 
- The possibility of Tier 3 administrative domain merger and split: investigation regarding AAA and general architecture design.

- Financial transfer architecture and integration to AAA: implementation and evaluation.

- Further performance tests: evaluation of mobility inside the network with regard to basic service set handover, possibly based on IEEE 802.11r.

Nevertheless as we have identified the main weak point to be scalability and related work research point to unsuitable medium access schemes, the main challenge remains unsolved. We assume that the reduction of bottlenecks in the medium around the Internet gateway (MPP) is most likely to be possible with a combination of time divided access, spatial frequency reuse and application of multiple channels.

\section{References}

1. Jorgenson, D.: Information technology and the US economy. American Economic Review, 1-32 (2001)

2. Colecchia, A., Schreyer, P.: ICT investment and economic growth in the 1990s: is the United States a unique case? A comparative study of nine OECD countries. Review of Economic Dynamics 5(2), 408-442 (2002)

3. Bhavnani, A., et al.: The Role of Mobile Phones in Sustainable Rural Poverty Reduction, p. 22 (retrieved November 2008)

4. Muente Kunigami, A., Navas Sabater, J.: Options to Increase Access to Telecommunications Services in Rural and Low Income Areas. World 1(2), 3-4 (2010)

5. Qiang, C., Rossotto, C., Kimura, K.: Economic impacts of broadband. Information and Communications for Development 2009: Extending Reach and Increasing Impact, 35-50 (2009)

6. Aker, J.: Does Digital Divide or Provide? The Impact of Cell Phones on Grain Markets in Niger (2008)

7. Jensen, R.: The Digital Provide: Information (Technology), Market Performance, and Welfare in the South Indian Fisheries Sector*. The Quarterly Journal of Economics 122(3), 879-924 (2007)

8. Marasinghe, V., Abeykoon, P., Wootton, R.: Strategies to promote ehealth and telemedicine activities in developing countries. Telehealth in the Developing World, 79 (2009)

9. Lucas, H.: Information and communications technology for future health systems in developing countries. Social Science \& Medicine 66(10), 2122-2132 (2008)

10. UNCTAD, Information Economy Report 2006: The Development Perspective. United Nations Publications (2006)

11. Williams, M.: Advancing the Development of Backbone Networks in Sub- Saharan Africa. Extending Reach and Increasing Impact, 51

12. Mbarika, V.: Re-thinking Information and Communications Technology Policy Focus on Internet versus Teledensity Diffusion for Africa's Least Developed Countries. EJISDC 9, $1-13$ (2002)

13. Knisely, D., et al.: Standardization of Femtocells in 3GPP. IEEE Communications Magazine, 69 (2009)

14. Lehr, W., McKnight, L.: Wireless internet access: $3 \mathrm{G}$ vs. WiFi? Telecommunications Policy 27(5-6), 351-370 (2003) 
15. Wellenius, B., Neto, I.: The radio spectrum: opportunities and challenges for the developing world. Info-Cambridge-Camford Publishing Limited 8(2), 18 (2006)

16. Garber, L.: Will 3G really be the next big wireless technology? Computer, 26-32 (2002)

17. Open-Mesh. Open-Mesh Webpage (2010), https : / /www. open-mesh.com/

18. Technologies, S.N.: Qualnet Product Webpage (2010), http: / / www.scalablenetworks.com/products / qualnet/ (cited 2010 05/11/2010)

19. UCLA, P.C.D., Global Mobile Information Systems Simulation Library (2010)

20. Gunasekaran, V., Harmantzis, F.: Emerging wireless technologies for developing countries. Technology in Society 29(1), 23-42 (2007)

21. Ishmael, J., et al.: Deploying rural community wireless mesh networks. IEEE Internet Computing, 22-29 (2008)

22. meraka.org. testbed networks (2010), http://wirelessafrica.meraka.org.za (cited 20.01.2010)

23. Barcelo, J., Oliver, M., Infante, J.: Adapting a Captive Portal to Enable SMS-Based Micropayment for Wireless Internet Access. In: Stiller, B., Reichl, P., Tuffin, B. (eds.) ICQT 2006. LNCS, vol. 4033, pp. 78-89. Springer, Heidelberg (2006)

24. Njenga, A.: Mobile phone banking: Usage experiences in Kenya

25. Hiertz, G., et al.: Principles of IEEE 802.11 s (2007)

26. Jun, J., Sichitiu, M.: The nominal capacity of wireless mesh networks. IEEE Wireless Communications magazine 10(5), 8-14 (2003)

27. Hiertz, G., et al.: IEEE 802.11 s: WLAN mesh standardization and high performance extensions. Language 890, 8044

28. Kyasanur, P., et al.: Multichannel mesh networks: challenges and protocols. IEEE Wireless Communications 13(2), 30-36 (2006) 